\title{
A Threat-Vulnerability Based Risk Analysis Model for Cyber Physical System Security
}

\author{
Lehlogonolo Ledwaba ${ }^{1,2}$ \\ ${ }^{1}$ Meraka Institute, Council for Scientific and \\ Industrial Research, Pretoria, 0184, South Africa \\ lehlogonolo.ledwaba@yahoo.com
}

\author{
H.S. Venter ${ }^{2}$ \\ ${ }^{2}$ Department of Computer Science, University of \\ Pretoria, Pretoria, 0083, South Africa \\ hventer@cs.up.ac.za
}

\begin{abstract}
The ability to network machinery and devices that are otherwise isolated is highly attractive to industry. This has led to growth in the use of cyber-physical systems (CPSs) with existing infrastructure. However, coupling physical and cyber processes leaves CPSs vulnerable to security attacks. A threat-vulnerability based risk model is developed through a detailed analysis of CPS security attack structures and threats. The Stuxnet malware attack is used to test the viability of the proposed model. An analysis of the Natanz system shows that, with an actual case security-risk score at Mitigation level 5, the infested facilities barely avoided a situation worse than the one which occurred. The paper concludes with a discussion on the need for risk analysis as part of CPS security and highlights the future work of modelling and comparing existing security solutions using the proposed model so to identify the sectors where CPS security is still lacking.
\end{abstract}

\section{Introduction}

In recent years, the advent of the Internet of Things has seen industrial applications moving from wired networking interfaces towards wireless and hybrid networking solutions. One such example is a cyberphysical system (CPS) which uses a combination of sensors, actuators, communication networks and cyber processing to facilitate learning, decision-making and self-actuation in multiple domains. CPSs tightly integrate cyber and physical processes; introducing new security challenges and vulnerabilities. With the increasing prevalence of cyber-crime, the protection of industrial and infrastructural networks is of an increasingly growing priority.

The development of efficient CPS security solutions is made difficult by the complexities and challenges unique to these networks. One example of this is the limited computing resources available to
CPS devices. Security solutions need to be able to account for the tight constraints and work efficiently and effectively without the complete depletion of available resources. It is therefore necessary to thoroughly investigate CPS architecture, the application domains in which a CPS may be utilised and the security challenges that need to be addressed in order to facilitate the development of specialised security solutions.

CPSs have security challenges different to those found in traditional IT systems. Attempts at mapping solutions from other sectors of networking such as sensor networks have been made with various degrees of success; however, as the solutions were not originally intended for CPSs, they often fail to meet all the security needs required by the system [23]. To this end, an analysis of the security holes occurring within CPSs is needed in order to identify in which areas a CPS is affected by a security attack and the extent of potential damage that may occur in the event of a successful attack

The main problem faced is in understanding the detailed operation interactions of the physical and cyber processes within a CPS and how they contribute to introducing security vulnerabilities. This understanding is necessary in order to develop successful security solutions that are able to provide maximum system security with minimum overhead. CPS security frameworks and architectures need to be to incorporate a variety of needs at the design level such as the precision required by the computing applications in a CPS for interfacing with the uncertainty and noise generated in a physical environment. For the context of this research, noise is defined as the random variation that may be present in measured values owing to interferences from the physical environment and the inherent manufacturing differences between components and devices. The occurrence of this variation leads to a percentage of error that is associated with the accuracy of each measurement. This error is defined as uncertainty. 
The remainder of the paper is organised as follows: Section 2 provides a detailed background on CPSs. Section 3 proposes a risk model based upon the threats and attack model identified in Section 2. Section 4 gives an implementation test of the model and the results thereof. Section 5 discusses the need for risk analysis as a necessary part of CPS security and identifies the contributions made towards CPS security by the proposed model. Section 6 concludes the paper and identifies the additional testing of the model's viability that shall be conducted as the model is improved upon as part of continuing work.

\section{Background}

Cyber-physical systems (CPSs) are a networked collection of "actuators, sensors, control processing units and communication devices" [4] designed to interface with physical and cyber processes to form part of a larger, distributed system [4], [19]. The operations in a CPS are aimed at monitoring the behaviours of the physical environment in order to achieve certain goals, such as effective resource allocation or early warning signalling, with actuation being based upon the results of computation processes performed at the cyber core [23]. The cyber core of a CPS consists of "computational availability that monitors, coordinates and controls the physical system" [2] and is found within the cyber domain.

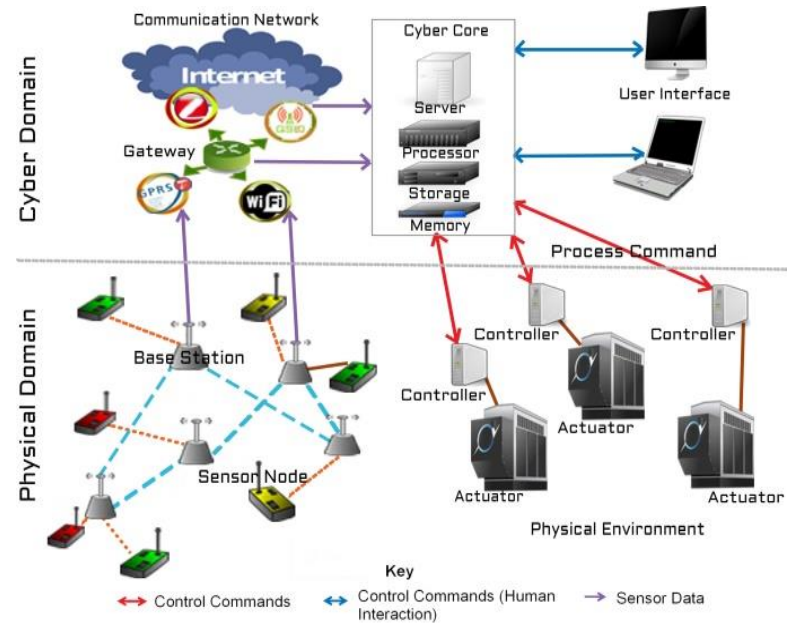

Figure 1: Cyber-physical system architecture

The unique operational structure of a CPS is detailed in Figure 1. Typically, a CPS utilises distributed control and management mechanisms in order to connect components into a networked configuration. Using a combination of wired and wireless communications, sensor data is sent into the cyber domain for concurrent processing and actuation
[22]. Computing results are then sent from the cyber core into physical components in order to effectively facilitate machine learning, system adaptability and network self-assembly and self-organisation [22]. This integration of physical and cyber processes indicated as red double arrows in Figure 1 - results in the predictable behaviour and real-time operation seen in CPSs.

The use of CPSs is becoming more prevalent in industry owing to the capability to network systems that are ordinarily isolated from the cyber core [3]. Examples of the CPS's application domains include smart resource and infrastructure management, emergency detection and response systems, smart health care and smart manufacturing infrastructure [16], [22]. The increase in prevalence of CPSs highlights the increasing priority for effective security solutions. As part of understanding the security needs of CPSs, a scalable, risk analysis and quantification model is needed in order to assist in the faster identification of high priority CPS vulnerabilities for which security solutions are required. Wu et al [25] proposed a risk quantification model for CPSs in 2015. The model specifies the security risks in CPSs and their interdependencies as vulnerability dependency graphs based upon the structure of directed acyclic graphs. The graphs are then utilised to calculate the system risk to illustrate which area(s) of the CPS would form the optimal attack target [25]. The problem with the use of acyclic graphs is that, as the number of threats and vulnerabilities found in a system increases, the graph size rapidly grows until it becomes too complicated for practical use. This limits the scalability of the model for larger industrial systems.

The threat-vulnerability risk model proposed by the authors in this paper is easily scalable and can be applied to either the individual sectors of a CPS such as the sensor or communication networks - or to a CPS as a whole unit without needing any implementation changes to the methodology and without the excessive growth of the model.

\section{CPS risk model}

The coupling of cyber and physical processes in a CPS exposes various security vulnerabilities and threats that may be utilised in the event of a cyberphysical attack. These attacks can be of a similar nature to traditional cyber-attacks and may be broken down into five main stages: access, discovery and reconnaissance, infiltration, damage and clean up [9]. From the analysis conducted in [8], [9], [25], and [26], the authors have identified five general-form CPS threats, based upon the general characteristics and behaviours exploited and the resultant damage caused 
to the system, as the foundation upon which the proposed model has been developed. These identified security threats are categorised as system behaviour alteration, denial of service, deception, network alteration and eavesdropping. The threats are seen to occur in the physical, communication and cyber components of the system and are categorised based upon the range of attacks with which the system may be faced along with the results thereof in the event of a successful attack.

In order to facilitate future, efficient development of critical CPS security solutions, the proposed model aims to provide a uniform methodology with which to identify the threats and vulnerabilities that need to be addressed urgently in addition to providing a quantitative scale against which one can compare the effectiveness of the developed solutions.

\subsection{Risk analysis variables}

As part of conducting a thorough risk analysis on a CPS, a variety of system characteristics are considered towards the calculation of the impact a threatvulnerability risk will have, overall, on the CPS. These characteristics were identified in accordance to the anatomy of a cyber-physical security attack and the security threats previously identified. The variables are chosen as the characteristics most often used by attackers in order to efficiently compromise a CPS.

The risk impact $\left(\mathrm{R}_{\mathrm{I}}\right)$ of a security threat or vulnerability is defined as the resultant damage to a CPS owing to the successful exploitation of a security threat or vulnerability. For a CPS threat/vulnerability, the risk impact is calculated with the consideration of the following factors:

- $\mathrm{E}_{\mathrm{P}}$ : the entry point score. This score gives an overview of the degree of openness of the CPS.

- $A_{\mathrm{P}}$ : the physical access score. This score quantifies the time needed for infiltration using entry points leading into the physical domain of the CPS or through physical intrusions by humans into the system.

- $\quad \mathrm{A}_{\mathrm{C}}$ : the cyber access score. This score quantifies the time needed for infiltration using the entry points leading into the cyber domain of the CPS.

- $\mathrm{C}_{\mathrm{F}}$ : the consequence factor associated with the resultant damage caused during and after a successful attack. Calculation of the consequence factor is based upon the damage caused to the system, to the environment and to human life. The variables used in the calculation of the consequence factor $\left(\mathrm{C}_{\mathrm{F}}\right)$ are weighted in order to provide a translation of the severity of the damage considered.
- $\quad$ System damage $\left(D_{S}\right)$ considers results such as the mechanical damage done to the compromised component and other system components within the affected sector of the CPS as a result of a successful attack and the operational delay that may occur in the system.

- Environmental damage $\left(\mathrm{D}_{\mathrm{E}}\right)$ considers the damage that may occur to the surrounding ecosystems owing to unintended emissions from the facility utilising the CPS.

- Damage caused to human life $\left(D_{H}\right)$ considers the potential for injury or the loss of life as a result of the compromised CPS and is given the most weight. This variable considers the damage that could be done to operational personnel, system end users and bystanders.

- PSR: the potential for complete system recovery following a successful attack. This score is in line with the degree of clean up done by the attackers and the degree of clean up needed for the system to resume uninhibited operation.

\subsection{Risk scoring}

For the purposes of the proposed model, the risk scoring is primarily based upon the overall cost to the company utilising CPSs in the event of a successful attack. The cost may be calculated in terms of loss of operational time, time spent on the recovery of data, loss of data, sensed or stored, during system downtime and monetary costs. These costs could be accrued in the form of personnel pay-outs, clean-up operation costs, the cost of abandoning facilities in cases of irrecoverable damage or the cost of replacements and repairs to damaged physical systems.

The scales allocated for the model variables have been chosen for easier interpretation and can be changed to more appropriate scales in individual cases of risk analysis. However, it is important to observe the weights allocated to each variable to ensure that the total is split correctly amongst the variables. In the proposed risk model, the risk impact score is calculated as a total out of 100. This total is split between the four main variables $E_{P}, A_{P}, A_{C}$ and $C_{F}$. $C_{F}$, as the variable with the most weight amongst the four variables, has a total score double to that of the other three variables and is calculated as a score out of $40 . \mathrm{C}_{\mathrm{F}}$ is given double the weight of the entry and access variables as it is an indicator of the physical damage that can occur to the CPS; the most important of which is the damage that may occur to employees and bystanders. 
The remainder of the risk impact total is split evenly between $E_{P}, A_{P}$ and $A_{C}$ with a score out of 20 for each.

3.2.1. The entry points $\left(\mathbf{E}_{\mathbf{P}}\right)$ score. The $E_{P}$ score is determined by the total number of unsecured, potentially exploitable entry points into the CPS. Some examples of exploitable entry points are given below [11], [18]:

- Number of devices that have been infected.

- Number of development backdoors and holes in the network perimeter.

- Number of unsecured/forgotten infrastructure e.g. modems connected to physical machines, dial-up access to remote terminal units (RTUs), field equipment modems.

- Number of accessible connection hijacking points within network.

- Number of compromised, outsourced firmware, software and equipment.

- Number of 'trusted' ports of the system e.g. USB ports, Ethernet cards, battery monitors.

The score for the entry point variable is determined using proportional scoring. The minimum and maximum values are mapped along the scale of 0 to 20 where 0 is indicative of a completely secured system with no exploitable entry points and 20 is indicative of a completely open, unsecured system where all entry points are exploitable gateways into the system. A scale of 20 was chosen as a result of the division of the final risk score - given on a scale of 0 to 100 amongst its four variables. The authors note however that any scale may be utilised if applied uniformly across all the risk model variables and with the necessary adjustments being made to the maximum values for the PSR and risk impact variables.

In order to calculate the $\mathrm{E}_{\mathrm{P}}$ score, two additional metrics are required:

- $\mathrm{MAX}_{\mathrm{E}}$ : This is the total number of entry points into the CPS both secured and unsecured. This number is dependent on the size of the CPS under analysis.

- UNSEC $_{\mathrm{E}}$ : This is the total number of entry points into the CPS that are unsecured.

Using the proportion of $\mathrm{UNSEC}_{\mathrm{E}}$ to $\mathrm{MAX}_{\mathrm{E}}$, the $\mathrm{E}_{\mathrm{P}}$ score, as a result out of 20 , can be calculated by:

$$
\mathrm{E}_{\mathrm{P}}=\frac{U N S E C_{E}}{M A X_{E}} \times 20
$$

The use of proportional scoring aids in illustrating how close or far the system is from being completely open to exploitation. A score closer to the mapped minimum is indicative of a more closed, secure system while a score closer to the mapped maximum is indicative of a more accessible, vulnerable system.

Proportional scoring for the entry point count is also used in an attempt to try and generalise the differences between CPSs or within various sectors of a CPS, resulting in a wider range of applicability for the model. This is needed because the number of entry points may differ from system to system or the number may differ from sector to sector. Some systems may have a smaller number of system entry points while some may have a larger number of entry points as a result of the sizes of the systems. Proportional scoring allocates a score based on the proportion of the unsecured count against the relative maximum; allowing for the same degree of urgency to be conveyed by the $\mathrm{E}_{\mathrm{P}}$ score, regardless of the scale of the system.

3.2.2. The access ( $A_{P}$ and $\left.A_{C}\right)$ scores. The scores for the $A_{P}$ and $A_{C}$ variables are determined from the entry points identified for the calculation of $E_{P}$. For these variables, the entry points are further divided into the relevant systems - the physical or cyber systems that they primarily affect when exploited.

For the unsecure access points, the average time-tocompromise (TTC) would need to be determined. Multiplication of the average TTC by the number of identified access points would make up the intermediary scores of $\mathrm{TTC}_{\mathrm{P}}$ and $\mathrm{TTC}_{\mathrm{C}}$.

TTC is defined as the estimated time taken by an attacker of a particular skillset to successfully infiltrate a targeted system [17]. For the purposes of the $A_{P}$ and $A_{C}$ scores, the TTC should be estimated for an attacker of average skillset with access to standard tools and hardware however; the facility exists for the score to be determined for a more skilled attacker with access to more sophisticated tools.

A variety of techniques may be used in order to determine the TTC. Estimation techniques have been proposed by LeMay et al. [14], Leversage and Byres [15], and McQueen et al. [17]. These techniques may be used to determine the TTC of the access points. The TTC can also be determined as part of system testing by the testing engineers of the CPS.

As with the determination of the $E_{P}$ score, proportional scoring, on a scale of 0 to 20 , is used to determine the scores for $\mathrm{A}_{\mathrm{P}}$ and $\mathrm{A}_{\mathrm{C}}$. For both variables, zero time taken for a successful exploitation is mapped to 20 while the maximum time taken for a successful exploitation is mapped to 0 . The value for the maximum time is determined as the average time taken to brute force entry into the system.

In order to determine $A_{P}$ and $A_{C}$, the following metrics are utilised: 
- $\mathrm{N}_{\mathrm{P}}$ : the number of unsecured, physical system entry points. This number is a subset of the number of entry points identified as part of $\mathrm{UNSEC}_{\mathrm{E}}$.

- $\mathrm{N}_{\mathrm{C}}$ : the number of unsecured, cyber system entry points. This number is also a subset of the number of entry point identified as part of $\mathrm{UNSEC}_{\mathrm{E}}$.

- TTC $_{\mathrm{Pi}}$ : The average TTC for a physical system entry point.

- $\mathrm{TTC}_{\mathrm{Cj}}$ : The average TTC for a cyber system entry point.

- $\mathrm{TTC}_{\mathrm{P}}: \mathrm{TTC}_{P i} \times N_{P}$

- $\mathrm{TTC}_{\mathrm{C}}: \mathrm{TTC}_{\mathrm{Ci}} \times N_{C}$

- $\mathrm{MAX}_{\mathrm{Pi}}$ : The average brute force entry time for physical domain entry points.

- $\mathrm{MAX}_{\mathrm{Ci}}$ : The average brute force entry time for cyber domain entry points.

- $\mathrm{MAX}_{\mathrm{P}}: \mathrm{MAX}_{\mathrm{Pi}} \times N_{P}$

- $\mathrm{MAX}_{\mathrm{C}}: \mathrm{MAX}_{\mathrm{Ci}} \times N_{C}$

The scores for $\mathrm{A}_{\mathrm{P}}$ and $\mathrm{A}_{\mathrm{C}}$ are calculated as follows:

$$
\begin{aligned}
& \mathrm{A}_{\mathrm{P}}=20-\left(\frac{T T C_{P}}{M A X_{P}} \times 20\right) \\
& \mathrm{A}_{\mathrm{C}}=20-\left(\frac{T T C_{C}}{M A X_{C}} \times 20\right)
\end{aligned}
$$

In the case of the two variables defined above, proportional scoring gives a quantification of the ease with which an unsecured entry point can be compromised. Scores closer to twenty are indicative of entry points that are quickly compromised while scores closer to zero are indicative of entry points that are harder to compromise. One may find there are unsecured entry points that could, relatively, take longer to compromise in order to gain entry into the system as opposed to other entry points. The priority in securing these entry points could then be lower than the priority for the entry points that are more easily compromised and thus more likely to be exploited.

3.2.3. The consequence factor $\left(\mathbf{C}_{\mathbf{F}}\right)$. The consequence factor is made up of three variables: Damage to human life $\left(\mathrm{D}_{\mathrm{H}}\right)$, Environmental Damage $\left(D_{E}\right)$ and System Damage $\left(D_{S}\right)$, which contribute to a total score of $40 . \mathrm{D}_{\mathrm{H}}$ is given the largest weight and constitutes $50 \%$ of the $\mathrm{C}_{\mathrm{F}}$ total. It is allocated along a scale of 0 to $20 . \mathrm{D}_{\mathrm{E}}$ is given the second-highest weight and constitutes $30 \%$ of the $\mathrm{C}_{\mathrm{F}}$. It allocated a score along a scale of 0 to 12 , where a score of 0 reflects no environmental damage as a result of the security failure and a score of 12 reflects irreversible, irrecoverable environmental damage. $\mathrm{D}_{\mathrm{S}}$ is given the lowest weight and thus constitutes $20 \%$ of the $\mathrm{C}_{\mathrm{F}}$.
The $\mathrm{D}_{\mathrm{S}}$ score is allocated along a scale of 0 to 8 . Should no damage occur to the system, a score of 0 is to be given and severe, irreparable damage is allocated a score of 8 . The score allocation is based on the percentage of the system that is reparable and the percentage of the system that would be written off and replaced; the cost of which is borne by the company utilising the CPS. It can be seen from Table 1 that these percentages are the inverses of each other.

The scoring for $\mathrm{D}_{S}$ is given as follows.

\section{Table 1: Risk scoring for system damage $\left(D_{s}\right)$}

\begin{tabular}{|c|c|c|}
\hline $\begin{array}{c}\text { Percentage } \\
\text { Repairable }\end{array}$ & $\begin{array}{l}\text { Percentage } \\
\text { Written off }\end{array}$ & Risk Score \\
\hline $0 \%$ & $0 \%$ & 0.0 \\
\hline $100 \%$ & $0 \%$ & 0.5 \\
\hline $99-80 \%$ & $1-20 \%$ & 1.0 \\
\hline $79-60 \%$ & $21-40 \%$ & 2.0 \\
\hline $59-40 \%$ & $41-60 \%$ & 4.0 \\
\hline $39-20 \%$ & $61-80 \%$ & 6.0 \\
\hline $19-0 \%$ & $81-100 \%$ & 8.0 \\
\hline
\end{tabular}

The score for $D_{E}$ is allocated based on the associated cost of the damage caused to the environment as a result of the failure of a CPS in the event of a security attack. This cost is often determined by the international liability conventions for the various sectors in which CPSs are employed. An example of one such convention, within the nuclear energy sector, is the 1963 Vienna Convention on Civil Liability for Nuclear Damage [10].

The scale for $D_{E}$ is split into two categories in order to account for the majority of damage scenarios that may occur. The first half of the scale, 0 to 6 , accounts for cases in which combinations of no damage and repairable damage have occurred to the environment surrounding the facility. The second half of the scale, 7 to 12 , accounts for cases in which combinations of repairable and irreparable damage has occurred to the environment surrounding the CPS facility. The score allocations are summarised in the tables below.

Table 2: Environmental score for cases of repairable damage and no damage

\begin{tabular}{|c|c|c|}
\hline $\begin{array}{c}\text { Percentage } \\
\text { Repairable }\end{array}$ & $\begin{array}{c}\text { Percentage } \\
\text { Undamaged }\end{array}$ & Risk Score \\
\hline- & $\sim 100 \%$ & 0.0 \\
\hline $1-10 \%$ & $\sim 90 \%$ & 1.0 \\
\hline $11-20 \%$ & $\sim 80 \%$ & 2.0 \\
\hline $21-40 \%$ & $\sim 60 \%$ & 3.0 \\
\hline $41-60 \%$ & $\sim 40 \%$ & 4.0 \\
\hline $61-80 \%$ & $\sim 20 \%$ & 5.0 \\
\hline $81-100 \%$ & $0 \%$ & 6.0 \\
\hline
\end{tabular}




\section{Table 3: Environmental score for cases of repairable and irreparable damage}

\begin{tabular}{|c|c|c|}
\hline $\begin{array}{c}\text { Percentage } \\
\text { Repairable }\end{array}$ & $\begin{array}{c}\text { Percentage } \\
\text { Irreparable }\end{array}$ & Risk Score \\
\hline$\sim 90 \%$ & $1-10 \%$ & 7.0 \\
\hline$\sim 80 \%$ & $11-20 \%$ & 8.0 \\
\hline$\sim 60 \%$ & $21-40 \%$ & 9.0 \\
\hline$\sim 40 \%$ & $41-60 \%$ & 10.0 \\
\hline$\sim 20 \%$ & $61-80 \%$ & 11.0 \\
\hline$\sim 0 \%$ & $81-100 \%$ & 12.0 \\
\hline
\end{tabular}

$\mathrm{D}_{\mathrm{H}}$ is allocated a score along the scale of 0 to 20 ; with 0 indicating no occurrences of injury and a score of 20 indicating fatal injury to nearly all employees.

The score allocated to $\mathrm{D}_{\mathrm{H}}$ is based upon the overall cost of compensation per employee injured in the event of a system failure. The compensation amount for occupational injury and disease differs from country to country and this calculation would need to be done in accordance with the compensation policies used per country.

In the same manner that the $\mathrm{E}_{\mathrm{P}}, \mathrm{A}_{\mathrm{C}}$ and $\mathrm{A}_{\mathrm{P}}$ scores are calculated; $\mathrm{D}_{\mathrm{H}}$ can be given as follows:

Where:

$$
\mathrm{D}_{\mathrm{H}}=\frac{T C_{E I}}{M A X_{T C}} \times 20
$$

- $\mathrm{TC}_{\mathrm{EI}}$ : Total compensation cost for employees actually injured. This is calculated by (the number of employees affected) $\mathrm{x}$ (average compensation per employee per annum).

- MAX $_{\mathrm{TC}}$ : Total compensation cost for all employees (injured and not). This is calculated by (the total number of employees) $\mathrm{x}$ (average compensation per employee per annum).

3.2.4. The potential for system recovery (PSR) score. Traditionally, business disaster management techniques include the definitions of a Recovery Time Objective (RTO) and Recovery Point Objective (RPO) in order to set the recoverability standard required by the organisation [21]. The PSR score serves to provide a comparison point for a CPS's actual capacity to recover from a disaster against the set RTO and RPO conditions as well as serving as a risk mitigation factor that reduces the overall impact of the security risk. As a result, the same scale used for the risk impact scoring is utilised for the PSR score.

The PSR score is allocated along a scale of 0 to 100 , where systems with the potential to make a recovery with no loss of resources are allocated the highest score of 100 and where systems with no potential of recovery and a complete loss of resources are allocated the lowest score of 0 . For the purpose of the PSR score, recoverability is defined as the percentage of the system operations that can be recovered after a security attack within the predetermined, allowed operation downtime period. If the PSR score of a security vulnerability or threat is high, then the risk associated with an exploitation of that security weakness is negated.

A full breakdown of the PSR score allocation scale is given in Table 4.

Table 4: Potential for system recovery (PSR) scoring

\begin{tabular}{|c|c|c|}
\hline $\begin{array}{c}\text { Percentage } \\
\text { recovery (\%) }\end{array}$ & PSR score & $\begin{array}{c}\text { Severity } \\
\text { Indicator }\end{array}$ \\
\hline 0 & 0.0 & No recovery \\
\hline $1-10$ & $1.0-10.0$ & Very little recovery \\
\hline $11-20$ & $11.0-20.0$ & \\
\hline $21-30$ & $21.0-30.0$ & \\
\hline $31-40$ & $31.0-40.0$ & \\
\hline $41-50$ & $41.0-50.0$ & Moderate recovery \\
\hline $51-60$ & $51.0-60.0$ & \\
\hline $61-70$ & $61.0-70.0$ & \\
\hline $71-80$ & $71.0-80.0$ & \\
\hline $81-90$ & $81.0-90.0$ & \\
\hline $91-99$ & $91.0-99.0$ & Near full recovery \\
\hline 100 & 100.0 & Full recovery \\
\hline
\end{tabular}

\subsection{Risk quantification}

Taking into consideration the identified risk variables and the proposed scoring methodology, the risk associated with a security threat or vulnerability may be quantified using the analysis methodology given below.

The $\mathrm{C}_{\mathrm{F}}$ of a security weakness, as a score out of 40 , can be calculated by:

$$
\mathrm{C}_{\mathrm{F}}=\mathrm{D}_{\mathrm{S}}+\mathrm{D}_{\mathrm{E}}+\mathrm{D}_{\mathrm{H}}
$$

The risk impact $\left(\mathrm{R}_{\mathrm{I}}\right)$, as a score out of 100 , is then given as:

$$
\mathrm{R}_{\mathrm{I}}=\mathrm{E}_{\mathrm{P}}+\mathrm{A}_{\mathrm{P}}+\mathrm{A}_{\mathrm{C}}+\mathrm{C}_{\mathrm{F}}
$$

In order to quantify the severity of a security threat or vulnerability, $R_{I}$ is weighted against a probability factor $(\mathrm{P})$ between 0.0 and 1.0. This is in consideration of cases where the probability of occurrence may be low, even when the resultant risk score for a threat or vulnerability may be high and vice versa. Such cases may be the result of a number of varying reasons. One example is the need for a highly specialised skillset in the development of the potential malware. 
Allocation of the probability score needs to consider the environmental trends in the Information Security sector and the frequency and success rate of attacks on CPSs. An organisation's attack desirability also needs to be taken into close consideration. Some sectors in industry are more desirable to attackers, such as government-run facilities, and could have a higher probability of a security attack occurring.

The security risk score $\left(\mathrm{SR}_{\mathrm{S}}\right)$ for a CPS security threat or vulnerability can then be defined as:

$$
\mathrm{SR}_{\mathrm{S}}=\mathrm{P} \times\left[\mathrm{R}_{\mathrm{I}}-\mathrm{PSR}\right]
$$

In weighting the combined risk-recovery score against a probability score, a facility to associate urgency for the need of a successful security solution to a security threat/vulnerability is given. A top down approach may then be utilised, resulting in the faster development of solutions able to secure the most critical sectors in a CPS.

\subsection{Interpreting the risk score}

The calculated risk score can be given as either a positive (+) or negative (-) number. A positive score is indicative of a risk that has not been mitigated by the system's recoverability potential. In this case, the risk impact is greater than the system recoverability score and is interpreted against the Security Risk levels. A negative score is indicative of risk that has been mitigated by the system's recoverability potential. This is a case where the system recoverability score is greater than the risk associated with the vulnerability or threat. These scores are interpreted against the Risk Mitigation levels.

In order to determine the priority scales associated with the security risk and the risk mitigation, five CPS security attack cases are considered. The worst case scenario, $\left(R_{I}=100\right)$, is a case in which all the variables are given the maximum scores possible for their associated scales. The best case scenario, $\left(\mathrm{R}_{\mathrm{I}}=0\right)$, is a case in which all the variables are given the minimum scores possible for their associated scales. The middle case scenario, $\left(R_{I}=50\right)$, is a case in which all the variables are all set to the middle of their associated scales. The lower, $\left(R_{I}=25\right)$, and upper, $\left(R_{I}=75\right)$, quarter cases have their variable scores set along $25 \%$ and $75 \%$ of their scales, respectively.

By varying the PSR score in increasing intervals of $10 \%$ and the probability in increasing intervals of 0.25 , the resultant scores were analysed and grouped together to form five priority and mitigation levels. The resultant priority scale is proposed with the levels of severity and mitigation being indicated in descending order:
Table 5: Priority scale for security-risk and risk mitigation

\begin{tabular}{|l|l|l|l|l|}
\hline \multicolumn{2}{|l|}{$\begin{array}{l}\text { Security Risk } \\
\text { levels }\end{array}$} & $\begin{array}{l}\text { Score } \\
\text { Range }\end{array}$ & \multicolumn{2}{l|}{$\begin{array}{l}\text { Risk Mitigation } \\
\text { levels }\end{array}$} \\
\hline & Priority 1 & $60-100$ & & Mitigation 1 \\
\hline & Priority 2 & $40-60$ & & Mitigation 2 \\
\hline & Priority 3 & $20-40$ & & Mitigation 3 \\
\hline & Priority 4 & $8-20$ & & Mitigation 4 \\
\hline & Priority 5 & $0-8$ & & Mitigation 5 \\
\hline
\end{tabular}

Five priority levels are defined for the scores categorised as Security Risk.

Priority 1 risks are defined as Near Catastrophic or Catastrophic failure risks where the vulnerability or threat has a very high probability of successful exploitation. Little to no recoverability would be possible in the event of a security attack with a very high probability of the occurrence of fatalities in the event of system failure.

Priority 2 risks are defined as Serious failure risks where the vulnerability or threat has an above average chance of successful exploitation. Minor system recovery would be possible. The potential damage caused by the system failure is high with a high probability of serious, bordering life threatening, injury to the users and system bystanders.

Similar to Priority 1 and 2 risks, Priority 3 to 5 risks are defined respectively as Moderate failure risks, Minor failure risks, and Near to no or no failure risks.

For the risk mitigation, five levels have also been defined, detailing the degree to which the risk associated with the threat/vulnerability has been mitigated.

Mitigation level 1 is defined as Near to complete or complete risk mitigation. In this case, the risk associated with the vulnerability or threat is completely mitigated by the system recoverability and there exists little to no chance of the security weakness moving into a security risk priority level should the analysis conditions change.

Mitigation level 2 is defined as High risk mitigation where the majority of the risk associated with the vulnerability or threat is mitigated by the system recoverability. Here, there also exists very little chance of the security weakness moving into a security risk priority level.

Similar to Mitigation levels 1 and 2, Mitigation levels 3 to 5 are defined respectively as Moderate risk mitigation. Minor risk mitigation and Near to no risk mitigation. 


\section{Model testing}

In order to test the effectiveness of the developed risk model, a case study analysis of the well-known CPS attack called the Stuxnet worm has been conducted in order to try and assess the risk associated with the vulnerabilities exploited.

Discovered in 2010, the Stuxnet worm targeted the control systems at an Iranian uranium refinery, Natanz [5]. Infiltration of the worm into the network occurred through the unprotected USB drives on controller PCs and infestation was propagated to other PCs through other unprotected network devices which supported USB drives, such as printers and scanners [6]. The Stuxnet worm was developed to target the programmable logic controllers (PLCs) utilised within the refinery. PLCs are specialised computing devices with sensor capability that controls "automated physical processes" [5]. In the case of the Iranian nuclear facilities, the PLCs were responsible for controlling the rotational speed of the centrifuges [6].

The results of various forensic investigations reported the following on the effects of Stuxnet:

- A total of approximately 100,000 total hosts were infected by Stuxnet, $58.31 \%$ of which were situated in Iran [7].

- Approximately 12,000 initial infections could be traced back to five Iranian organisations [7].

- The shortest time between compile and infection time was 12 hours, the longest time was 28 days and the average time was 19 days [7].

- Of the 9,000 centrifuges deployed at the Natanz facility, about 1,000 of the centrifuges were decommissioned. The proximity of the decommission date to the discovery of the Stuxnet worm has led to the conclusion that the centrifuges were damaged as a result of Stuxnet, though it was not officially confirmed [1].

- Owing to a shortage of raw material, Iran only had the facility to build $12,000-15,000$ centrifuges. With the loss of 1,000 centrifuges to routine operational breakdowns, the additional loss of 1,000 to Stuxnet was significant [1].

- Recovery from the effects of Stuxnet took the Natanz facility approximately 6 months to accomplish [24].

With consideration of the vulnerabilities and threats exploited by the Stuxnet worm and the reported effects of the worm, a risk analysis of the infected CPS can be conducted through the application of the developed risk model.
Table 6: Risk analysis of the Natanz CPS

\begin{tabular}{|c|c|c|}
\hline $\begin{array}{l}\text { Risk } \\
\text { Variable }\end{array}$ & Calculation & Score \\
\hline $\begin{array}{l}\mathrm{E}_{\mathrm{P}} \\
{[0.0-20.0]}\end{array}$ & $\mathrm{E}_{\mathrm{p}}=\frac{58,310}{100,000} \times 20$ & 11.662 \\
\hline $\begin{array}{l}\mathrm{A}_{\mathrm{P}} \\
{[0.0-20.0]}\end{array}$ & $\mathrm{A}_{\mathrm{P}}=20-\left(\frac{9.5 \text { days }}{14 \text { days }} \times 20\right)$ & 6.429 \\
\hline $\begin{array}{l}\mathrm{A}_{\mathrm{C}} \\
{[0.0-20.0]}\end{array}$ & $\mathrm{A}_{\mathrm{C}}=20-\left(\frac{9.5 \text { days }}{14 \text { days }} \times 20\right)$ & 6.429 \\
\hline $\begin{array}{l}\mathrm{D}_{\mathrm{S}} \\
{[0.0-8.0]}\end{array}$ & $10 \%$ damage $=\frac{1,000}{9,000} \times 100$ & 1.0 \\
\hline $\begin{array}{l}\mathrm{D}_{\mathrm{E}} \\
{[0.0-12.0]}\end{array}$ & No known damage & 0.0 \\
\hline $\begin{array}{l}\mathrm{D}_{\mathrm{H}} \\
{[0.0-20.0]} \\
\end{array}$ & No known injuries or deaths & 0.0 \\
\hline $\begin{array}{l}\mathrm{P} \\
{[0.0-1.0]}\end{array}$ & Probability of reoccurrence & 0.40 \\
\hline $\begin{array}{l}\text { PSR } \\
{[0.0-} \\
100.0]\end{array}$ & $\begin{array}{l}\text { Recovered within } 6 \text { months but } \\
\text { utilised more of the limited raw } \\
\text { material to engineer an } \\
\text { additional } 1,000 \text { centrifuges [1], } \\
{[24]}\end{array}$ & 40.0 \\
\hline $\begin{array}{l}\mathrm{C}_{\mathrm{F}} \\
{[/ 40.0]}\end{array}$ & $\mathrm{C}_{\mathrm{F}}=\mathrm{D}_{\mathrm{S}}+\mathrm{D}_{\mathrm{E}}+\mathrm{D}_{\mathrm{H}}$ & 1.0 \\
\hline $\begin{array}{l}\mathrm{R}_{\mathrm{I}} \\
{[/ 100.0]}\end{array}$ & $\mathrm{R}_{\mathrm{I}}=\mathrm{E}_{\mathrm{P}}+\mathrm{A}_{\mathrm{P}}+\mathrm{A}_{\mathrm{C}}+\mathrm{C}_{\mathrm{F}}$ & 25.804 \\
\hline $\mathrm{SR}_{\mathrm{S}}$ & $\mathrm{SR}_{\mathrm{S}}=\mathrm{P} \times\left[\mathrm{R}_{\mathrm{I}}-\mathrm{PSR}\right]$ & -5.792 \\
\hline
\end{tabular}

It can be seen that an analysis of the Stuxnet infestation at Natanz, from the known information of its effects, results in a Mitigation 5 risk score. This is owing to the lack of environmental damage or incidents of injury or death. The score of 1.0 for system damage represents the interpretation of repairable damage with $10 \%$ of the damage written off. This is indicative of the required replacement of the ruined centrifuges, and the resultant loss of Iran's limited raw materials, and the continued operation of the facility. The infection time is equally divided between the physical and cyber access score calculations, as details on the individual compromise times are not specified. Although the risk associated with Stuxnet was mitigated, at Mitigation level 5; Natanz barely avoided an even more devastating result. Had the Stuxnet worm been programmed to cause more damage, or had the centrifuges failed in a more catastrophic manner, resulting in injury to the employees at Natanz or damage to the surrounding environment, the results of the risk analysis would have been within a Security Risk level, and thus become a matter needing more urgent attention in the Information Security domain.

As the situation stands, knowledge of the potential danger their systems faced to a devastating security attack could have encouraged better security practices at Natanz. Actions could have been taken to prevent 
the leakage of sensitive, facility layout and configuration information through the widely televised walkthrough tours, as analysed in [13], and best practice policies could have been implemented regarding the connection personal external devices such as USB sticks. In addition, awareness of the system's vulnerability to a security attack could have resulted in the earlier detection of the worm as a possible cause to the repeated damage to the centrifuges; reducing the overall effectiveness of the cyber-physical attack.

\section{Discussion}

Methods for risk quantification in CPS security are still a growing research area however, they are important as they provide the mechanism to identify the areas of high vulnerability, and consequently high risk, in a CPS in addition to assisting one in identifying the order in which solutions for CPS security need to be developed. In terms of the wider information security domain, risk analysis techniques and models aid in illustrating the need for digital forensic readiness strategies in an environment where, as the application of technology in everyday business operations continues to grow, so too does the threat of a major security breach.

The proposed model provides an overview of the system security that can be utilised during initial system design or when conducting risk evaluations. This allows the owners of the CPSs the opportunity to conduct thorough risk planning and decide which risks can be absorbed by the system, which risks can be mitigated or which risks can be outsourced to be handled by a third party. The proposed risk model also gives allowance for the development of forensic readiness and system recovery strategies for the eventuality of an attack. The results of this are better, more secure network designs. The simplicity of the model means that it can be integrated as part of scheduled CPS maintenance practices. This allows for the continuous monitoring of the state of the CPS's security, especially in cases were alterations are made to the system structure. The result is CPSs that are constantly secured from security attacks and are given allowance for the early detection of any changes to the security state. This could result in the quicker application of defensive measures on the system network and prevent damaging security attacks prior to their occurrence.

Apart from applications in CPSs, the proposed model can also be adjusted for use in the wider information security domain as part of the development of readiness strategies. Calculations involving the physical domain of a CPS can be interpreted as applying to the physical domain of an IT organisation allowing analysists to take into consideration the risks associated with damage as a consequence of forced entry into the organisation or cyber-attacks as a result of insider informants and perpetrators. The calculations surrounding damage to human life can be adjusted to consider the costs of employee retrenchment packages in the event of job losses as a result of a major security breach, to give one example.

More importantly, in proposing a simple and scalable risk analysis model, the authors aim to address two of the four major "security apocalypse horsemen" [20] namely security risk quantification and bridging the gap between security and business risk in addition to encouraging risk-driven specification in future CPS security solutions. Risk-driven specification is especially important in safety or mission critical CPSs as this allows developers to identify the states and conditions that must not be allowed to occur as a result of a security failure in addition to allowing for the increased survivability and resilience of CPSs, making them less attractive targets to malicious attackers [12].

The major problem facing the proposed model is one of subjectivity. The nature of risk analysis is that an element of subjectivity is always retained. Risk analysis is highly dependent on the bias of the person conducting the analysis and results could vary between analyses. While the model attempts to limit instances of subject bias through the use of independently, quantifiable metrics, it is still subjective to bias in the determination of the damage caused by system failure, the recovery potential of the system and the probability of the system coming under attack through the use of the identified threat or vulnerability.

\section{Conclusion}

It can be seen that in order for the development of successful CPS security solutions to occur, an understanding of the impact of a security failure is needed. Risk assessment models designed specifically for CPS security, such as the models proposed in this paper and in [25], are being developed with the focus on the threats and vulnerabilities that occur owing to the joining of cyber and physical processes. The proposed model is able to quantify the impact that a security failure could have on a system, adjust for recoverability in the event of a security failure and quantify the urgency needed for the development of solutions which address high risk threats and vulnerabilities. This allows for the easier identification of areas where CPS security solutions may be lacking.

As part of further work, a detailed analysis of additional CPSs shall be conducted in order to further verify the model's viability. An analysis of the current 
state of CPS security using the proposed model shall also be conducted in order to objectively compare the effectiveness of previously proposed solutions for securing CPSs and to identify areas where solution development has been lacking, so to begin work in developing a lightweight security framework for CPSs.

\section{References}

[1] David Albright, Paul Brannan, and Christina Walrond, "Stuxnet Malware and Natanz: Update of ISIS December 22, 2010 Report," Washington, 2011.

[2] PJ Antsaklis et al., "Control of Cyberphysical Systems using Passivity and Dissipativity Based Methods," European Journal of Control, vol. 19, no. 5, pp. 379-388, 2013.

[3] Radhakisan Baheti and Helen Gill, "Cyber-Physical Systems," The Impact of Control Technology, vol. 12, pp. 161-166, 2011.

[4] Alvaro A Cardenas et al., "Challenges for Securing Cyber Physical Systems," in Workshop on Future Directions in Cyber-Physical Systems Security, 2009, pp. 5-11.

[5] T.M Chen and S Abu-Nimeh, "Lessons from Stuxnet," Computer, vol. 44, no. 4, pp. 91-93, 2011.

[6] S Collins and S McCombie, "Stuxnet: The Emergence of a New Cyber Weapon and Its Implications," Journal of Policing, Intelligence and Counter Terrorism, vol. 7, no. 1, pp. 80-91, 2012.

[7] Nicolas Falliere, Liam O Murchu, and Eric Chien, "W32.Stuxnet Dossier Version 1.4," Cupertino, California, 2011.

[8] Y Gao et al., "Analysis of Security Threats and Vulnerabilities for Cyber-physical Systems," in 3rd International Conference on Computer Science and Network Technology, Dalin, China, 2013.

[9] Dieter Gollmann and Marina Krotofil, "Cyber-Physical Systems Security- An Experimental Approach," Hamburg University of Technology, Presentation.

[10] International Atomic Energy Agency. (2016) Vienna Convention on Civil Liability for Nuclear Damage. [Online].

https://www.iaea.org/publications/documents/conventions/ vienna-convention-on-civil-liability-for-nuclear-damage

[11] Stamatis Karnouskos. (2014, April) ERCIM News 97: Security in the Era of Cyber-Physical Systems of Systems. [Online]. http://ercimnews.ercim.eu/en97/special/security-in-the-era-of-cyberphysical-systems-of-systems

[12] Stan Kurkovsky, Software Engineering: Critical Systems Specification, Unknown, CS530 Lecture Slides- Central Connecticut State University.

[13] Ralph Langner, "To Kill a Centrifuge," The Langner Group, Hamburg, Technical Analysis 2013.
[14] Elizabeth LeMay et al., "Adversary-Driven State-Based System Security Evaluation," in MetriSec2010, BolzanoBozen, Italy, September 15, 2010.

[15] David John Leversage and Eric James Byres, "Estimating a System's Mean Time-to-Compromise," IEEE Security \& Privacy, vol. 6, no. 1, pp. 52-60, 2008.

[16] Tianbo Lu, Jinyang Zhao, Lingling Zhao, Yang Li, and Xiaoyan Zhang, "Towards a Framework for Assuring Cyber Physical System Security," International Journal of Security and its Applications, vol. 9, no. 3, pp. 25-40, 2015.

[17] Miles A McQueen, Wayne F Boyer, Mark A Flynn, and George A. Beitel, "Time-to-Compromise Model for Cyber Risk Reduction Estimation," in First Workshop on Quality of Protection, Quality of Protection: Security Measurements and Metrics.: Springer, 2005.

[18] Yilin Mo et al., "Cyber-Physical Security of a Smart Grid Infrastructure," Proceedings of the IEEE, vol. 100, no. 1, pp. 195 - 209, 2011.

[19] Ragunathan Rajkumar, Insup Lee, Lui Sha, and John Stankovic, "Cyber-Physical Systems: The Next Computing Revolution," Proceedings of the 47th Design Automation Conference, pp. 731-736, 2010.

[20] Rockwell Automation. (2015, March) Process Solutions Summit. [Online].

https://www.rockwellautomation.com/resources/download s/rockwellautomation/pdf/events/processsummit/CT522_Securing_Industrial_Networks.pdf

[21] Susan Snedaker, "Chapter 4: Business Impact Analysis," in Business Continuity and Disaster Recovery Planning for IT Professionals. Waltham, MA: Syngress, a division of Elsevier, 2007, pp. 218-225.

[22] Unknown, "An Introduction to Cyber-Physical Systems," University of Oslo, 30 Aug 2011.

[23] Eric Ke Wang et al., "Security Issues and Challenges for Cyber Physical Systems," in 2010 IEEE/ACM International Conference on Green Computing and Commincations \& 2010 IEEE/ACM International Conference on Cyber, Physical and Social Computing, 2010, pp. 733-738.

[24] Joby Warrick. (2011, February) Iran's Natanz nuclear facility recovered quickly from Stuxnet cyberattack. [Online]. www.washingtonpost.com/wpdyn/content/article/2011/02/15/AR2011021505395.html?s $\mathrm{id}=$ ST2011021404206

[25] W Wu, R Kang, and Z Li, "Risk Assessment Method for Cybersecurity of Cyber-Physical Systems Based on InterDependency of Vulnerabilities," in 2015 IEEE International Conference on Industrial Engineering and Engineering Management (IEEM), Singapore, 2015.

[26] F Xie et al., "Security Analysis on Cyper-Physical System Using Attack Tree," in 2013 Ninth International Conference on Intelligent Information Hiding and Multimedia Signal Processing, Beijing, 2013. 